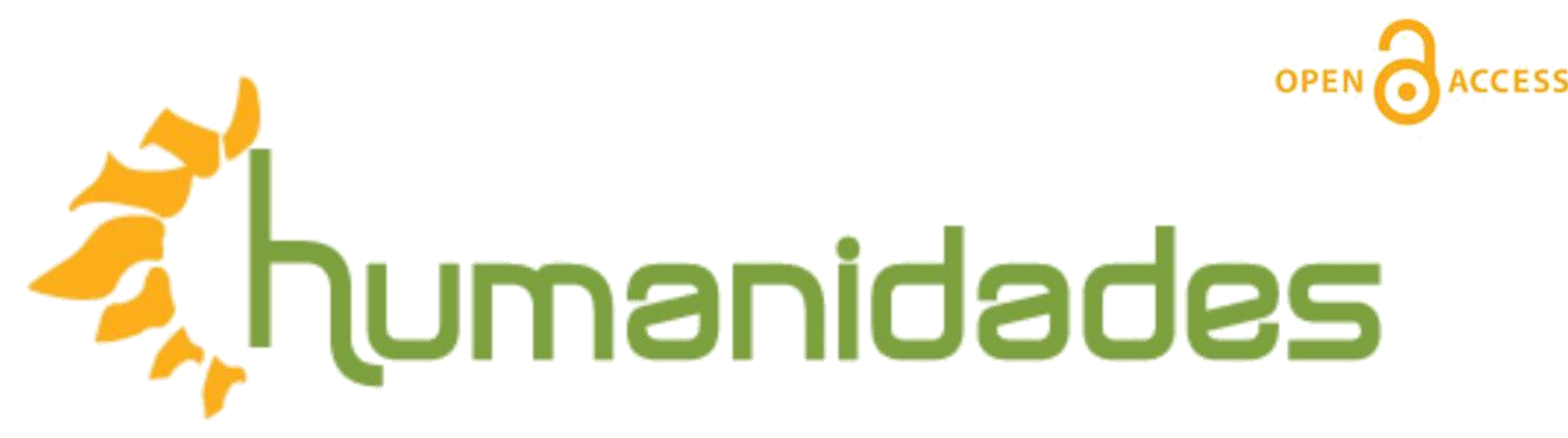

Revista de la Escuela de Estudios Generales, Universidad de Costa Rica

Julio-diciembre, 2017 • Volumen 7, número 2 • EISSN 2215-3934•

Recibido: 12- Marzo-2017 Aceptado: 15- Mayo- 2017

\title{
Ciencia y tecnología en América Latina: Una aproximación desde la Historia de la Cultura
}

DOI: http://dx.doi.org/10.15517/h.v7i2.29579

\section{Ronald Eduardo Díaz Bolaños}

Escuela de Estudios Generales y Centro de Investigaciones Geofísicas. Universidad de Costa Rica. COSTA RICA

Correo electrónico: ronald.diaz@ucr.ac.cr

\footnotetext{
Todos los derechos reservados. Universidad de Costa Rica. Esta revista se encuentra licenciada con Creative Commons. Reconocimiento-NoComercial-SinObraDerivada 3.0 Costa Rica.

Correo electrónico: humanidades@ucr.ac.cr / Sitio web: http://revistas.ucr.ac.cr/index.php/humanidades
} 


\section{Ciencia y tecnología en América Latina: Una aproximación desde la Historia de la Cultura}

\section{Resumen}

Este trabajo plantea una propuesta metodológica de cómo podría abordarse el papel de la ciencia y la tecnología en América Latina en el marco de una lección de Historia de la Cultura, basada en la experiencia docente del autor

Palabras claves: Ciencia, tecnología, América Latina, Historia Social de la Ciencia, docencia

\section{Science and technology in Latin America: An approach from the History of Culture}

\footnotetext{
Abstract

This paper presents a methodology of how it could be addressed the role of science and technology in Latin America as part of a lesson of Cultural History based on teaching experience of the author.

Keywords: Science, Technology, Latin America, Social History of Science, Teaching.
} 


\section{Introducción}

Las lecciones magistrales han sido el medio tradicional a través del cual se han impartido las lecciones de Historia de la Cultura en la Escuela de Estudios Generales de la Universidad de Costa Rica, si bien se han dado cambios en la metodología de la enseñanza de este curso, por ejemplo en los último años, parte del personal docente lo ha ido complementando con el aporte de material audiovisual o bien mediante los trabajos en grupo u otras técnicas empleadas para abordar los contenidos del programa oficial del curso. No obstante, se priorizan los contenidos que están relacionados con temáticas referentes a los aspectos contemporáneos, en su dimensión política, económica, social y cultural.

El programa del curso de Historia de la Cultura perteneciente al Curso Integrado de Humanidades Opción Regular II, que se imparte normalmente en el segundo ciclo de cada año, lleva como título "Los grandes retos de América Latina", aborda la actual realidad latinoamericana desde una perspectiva histórica mediante el análisis de diversos temas relacionados con la política, la economía, la sociedad y la cultura de la región enfocados desde una visión de conjunto.

Dentro de esa amplia gama de temas, existe un contenido que se denomina "El desafío científico y tecnológico", en el que se enfoca el impacto que ha tenido la ciencia y la tecnología en el desarrollo de las sociedades latinoamericanas. Esta temática puede abordarse desde distintos enfoques: Historia de la Ciencia (internalista), Historia Social de la Ciencia y la Tecnología (externalista), Estudios Sociales sobre Ciencia, Tecnología y Sociedad (CTS) o Estudios Sociales sobre Ciencia, Tecnología, Sociedad e Innovación (CTS +I).

En el presente trabajo, se analiza una experiencia docente desarrollada para abordar el tema de "El desafío científico y tecnológico" en una clase del curso Historia de la Cultura con base en el enfoque de Historia Social de la Ciencia.

\section{Aspectos conceptuales}

La Historia Social de la Ciencia es un enfoque que analiza el desarrollo científico en relación con el contexto político, económico, social y cultural en el que este se produce. De acuerdo con Saldaña (1996b, pp. 7-8), este enfoque:

pretende llegar a entender la naturaleza y el comportamiento que han seguido los grupos sociales (los científicos) que crean, desarrollan o incorporan conceptos y teorías en un contexto social particular y como consecuencia del mismo. Se presta atención igualmente a aquellos aspectos generales de la sociedad y de la geografía regionales (orden social, cultura, recursos naturales, posiciones geográficas, etcétera) que son responsables de las actitudes grupales hacia la ciencia y que le han impuesto un estilo particular.

Debido a la relación que existe entre el conocimiento y la actividad científica con el contexto en que se produce, a dicho enfoque se le ha considerado externalista, por lo que se contrapone con la visión internalista que caracteriza a la Historia de la Ciencia. Este último enfoque analiza el desenvolvimiento histórico de la ciencia como el resultado de una serie de cambios que se producen al interior de las disciplinas científicas, producto de las ideas y la labor de la comunidad científica que se desarrollan independientemente del contexto histórico en el que tienen lugar (Medina, 1983). 
En el caso de América Latina, la Historia Social de la Ciencia ha permitido analizar la construcción de las comunidades científicas (Azuela y Saldaña, 1994; Azuela, 1996b, Azuela y Guevara, 1998 y RodríguezSala, 2004), el papel de las exploraciones geográficas en el reconocimiento de los territorios que constituyen América Latina (Rodríguez-Sala, 2002a) y los procesos de institucionalización de disciplinas científicas como la astronomía (Rodríguez-Sala, 2002b) y la meteorología (Azuela, 1995; Solano, 1999 y Díaz, 2003), así como de las ciencias en general (D’Ambrosio, 1986 y Azuela, 1996a). También, esta corriente ha propuesto enfoques teóricos que explican los factores políticos, económicos y culturales que favorecen el desarrollo científico en algunos países de la región (Clare y Viales, 2006-2007 y 2009).

Además, desde este enfoque se han construido periodizaciones para explicar el desarrollo históricocientífico latinoamerciano, como la propuesta por Hebe Vessuri (1996) que se compone de cinco etapas resumidas en el cuadro 1:

\section{Tabla 1}

Periodización del desarrollo histórico-científico latinoamericano según Vessuri (finales siglo XIX hasta 1990)

Período

Finales del siglo XIX y principios del siglo $\mathrm{XX}$
Período de la ciencia positivista: Estado incipiente de las comunidades científicas, influencia del positivismo, exploraciones de reconocimiento de los territorios y sus recursos naturales, primeras instituciones científicas y debates con científicos de otras latitudes.

1918-1940

Institucionalización de la ciencia experimental: Consolidación de las instituciones científicas, modernización de las universidades y promoción de la cooperación internacional con organismos científicos extrarregionales.

1940-1960

Décadas de desarrollo: Políticas desarrollistas promueven vínculos mayores entre la ciencia y las universidades y surgimiento de nuevas instituciones científicas.

1960-1980

Edad de la política científica: Fundación de instituciones y consejos científicos gubernamentales, amplio respaldo estatal a la investigación científica.

1980-1990

Etapa del empresariado industrial: mayor interés del sector empresarial en la actividad científica en sintonía con las políticas neoliberales en detrimento del apoyo estatal. 
La periodización de Vessuri finaliza en el año 1990, precisamente cuando estaban en marcha la aplicación de las políticas neoliberales en la mayor parte de los países latinoamericanos (Martínez y Soto, 2012), por lo que la hegemonía del empresariado industrial -y del sector privado en general- en la actividad científica, podría extenderse todavía una década más.

A partir del 2000, la llegada al poder de gobiernos de izquierda, inspirados en mayor o menor medida por el denominado Socialismo del Siglo XXI (Hamburguer, 2014), en algunos países de la región como Venezuela, Argentina, Brasil, Bolivia y Ecuador, podría ser el punto de partida de una sexta etapa del desarrollo histórico-científico regional basada en un mayor intervencionismo estatal en la economía y un nuevo impulso de la actividad científica desde el Estado en América Latina. Esta etapa podría extenderse hasta el 2015, fecha en que algunos de esos gobiernos comienzan a ser relevados por otros de tendencia neoliberal como en el caso argentino y brasileño (Margariti, 2016), lo que podría traer sus consecuencias en el desarrollo científico latinoamericano en los próximos años.

La periodización de Vessuri deja de lado los aportes de las civilizaciones de los pueblos originarios de América al desarrollo científico regional y universal (Valdivia, 1996), además de las experiencias científicas que tuvieron lugar en la región durante el período colonial, como por ejemplo, las expediciones ilustradas en el siglo XVIII (Lafuente y López-Ocón, 1996) y las primeras sociedades científicas que florecieron en las capitales virreinales al calor del movimiento ilustrado (Saldaña, 1996a).

A pesar de las limitaciones, la periodización de Vessuri es útil para comprender el desarrollo históricocientífico de América Latina entre finales del siglo XIX y la mayor parte del siglo XX, que coincide significativamente con el período en que se aborda el contenido sobre ciencia y tecnología abordado desde la experiencia docente que se explica a continuación.

Cabe señalar que, al ser un curso de Historia, se mantiene la dimensión cronológica propuesta por Vessuri, en el que se enmarca el desarrollo de la experiencia docente basada en el círculo de conversación, especialmente en la sesión de conversación general donde la dinámica de discusión de las preguntas asignadas a cada subgrupo, respeta dicho orden cronológico.

\section{Desarrollo de una experiencia docente en torno al desafío científico y tecnológico en América Latina}

Debido a la amplitud del contenido titulado "El desafío científico y tecnológico" que se incluye en el programa de Historia de la Cultura correspondiente al Curso Integrado de Humanidades Opción Regular II, este puede desarrollarse a través de una técnica como la del círculo de conversación. Esta técnica facilita la construcción del conocimiento mediante el aprendizaje colaborativo por parte de los y las estudiantes a partir del intercambio de experiencias relacionadas con el contenido de investigación, con el fin de analizar el papel que ha tenido la ciencia y la tecnología en el desarrollo de los países de América Latina desde una perspectiva histórica.

El propósito del aprendizaje es que los y las estudiantes interpreten en forma contextualizada el tema del aporte de la ciencia y la tecnología en el desarrollo de América Latina, mediante la explicación de dicho aporte y su importancia para los países de la región. Se trata de un tema de mucha actualidad y ha tenido una trayectoria histórica que se remonta a los tiempos anteriores a la conquista ibérica, estuvo presente durante el período colonial y ha tenido un desarrollo incipiente desde el siglo XIX hasta alcanzar una importancia trascendental en el presente, en el marco de la actual Revolución Científica y Tecnológica, donde los conocimientos científicos y los artefactos tecnológicos se han incorporado en la vida cotidiana de los seres humanos. 
La técnica del círculo de conversación permite a la clase compartir su conocimiento relacionado con el tema de la ciencia y la tecnología y su relevancia en el desarrollo de América Latina. El abordaje se realiza desde un punto de vista histórico mediante un aprendizaje colaborativo que favorece la construcción del conocimiento en forma conjunta.

El fundamento de esta técnica de acuerdo con Martínez (2010, citado por Arias, 2012), es el siguiente:

"se argumenta en la sabiduría de grupos y tradiciones indígenas de diferentes partes del mundo y en los conocimientos de las teorías dialógicas, de la resolución pacífica de conflictos, de la educación popular, de la educación holística y de la teoría de la dinámica de grupos" (p. 12).

Esta técnica había sido planteada en la experiencia desarrollada por Pranis, Stuart y Wedge (2003) y en el estudio de la Comisión Nacional para el Mejoramiento de la Administración de la Justicia de Costa Rica (CONAMAJ), desde donde se ha empleado para la formación y utilización de los círculos de conversación en diferentes contextos educativos, de organizaciones comunitarias, gobiernos locales y del sector corporativo (Bernal y Castillo, 2006, citado por Arias, 2012).

El propósito de utilizar esta técnica como estrategia didáctica, según Pranis et ál. (2003, citado por Arias, 2012) se fundamenta en el estímulo de la reflexión personal y colectiva, mediante la organización de actividades que permiten a las personas participantes expresarse libremente y a su vez ser escuchadas, sin que necesariamente se obtenga el logro de un objetivo en concreto- El fin primordial de esta técnica es buscar que las personas participantes aprendan de las demás y puedan mejorar sus relaciones sociales a través del conocimiento construido en forma colectiva.

De acuerdo con Arias (2012): "La utilización del círculo de conversación como estrategia didáctica posibilita diferentes formas de abordar un tema específico, mediante la significación y búsqueda de sentido, en las construcciones de conocimiento, de habilidades y de actitudes socializadas" (p. 13). De esta forma, mediante la libre participación de las personas, se va creando un ambiente de intercambio de ideas y conocimientos que en el campo de la Historia facilitaría una mayor comprensión de los procesos históricos a través del intercambio de ideas relacionadas con dichos procesos y en el caso específico del contenido seleccionado, sobre el papel que ha tenido la ciencia y la tecnología en el desarrollo económico y social de América Latina.

La técnica es posible aplicarla en un contexto espacial caracterizado por la presencia de un aula lo suficientemente amplia para un grupo de cuarenta a cuarenta y cinco estudiantes, donde se puede conformar un círculo para su desarrollo. Además, a lo largo del semestre, la población estudiantil asiste al curso de Historia de la Cultura con regularidad y muestra interés por las lecciones que se imparten.

Los alumnos y las alumnas tienen una activa participación en esta estrategia, por lo que debe integrarse a un subgrupo que será el mismo en que participa en el marco del proceso de investigación de la Guía Académica, por lo que ya se conoce a las personas con las que va a realizar el trabajo. Además del conocimiento que se ha adquirido con anterioridad en relación con este tema, se asigna la lectura de varios textos referentes a la experiencia científica y tecnológica en América Latina desde un punto de vista histórico.

Los y las estudiantes deben leer primero los textos asignados, uno por subgrupo, para comprender los procesos históricos relacionados con un aspecto del acervo científico y tecnológico latinoamericano, por lo que podrán debatir dicho aspecto con sus compañeros y compañeras de subgrupo a partir de la lectura, del conocimiento previo que tengan del tema y guiados por una pregunta relacionada con la lectura. Este debate se hará en igualdad de condiciones con respecto al resto de integrantes de los respectivos subgrupos de investigación dentro del aula. 
De esta forma, el círculo de conversación se organiza una vez que los miembros de los seis subgrupos de investigación en los que se ha dividido la clase, terminan la discusión y se forma un gran círculo donde tienen completa libertad de expresar sus ideas en relación con el aspecto que les tocó discutir relacionado con la ciencia y la tecnología en América Latina, en un marco de respeto en que participan cuando se les da la palabra, de acuerdo con el orden en que se organice la conversación. De esta forma el estudiantado se involucra en un proceso de aprendizaje colaborativo que le permitirá compenetrarse con el tema en forma colectiva, para luego pasar a debatirlo al final de la sesión en el que se comentarán las ideas principales del aspecto en que han debatido.

El docente en este caso se constituye en un facilitador del proceso para la población estudiantil que conforma la clase, en cuanto asigna las lecturas relacionadas con varios tópicos del desarrollo históciocientífico latinoamericano (exploraciones geográficas, institucionalización de disciplinas científicas, publicaciones periódicas, políticas públicas tecnocientíficas y percepción de la ciencia y la tecnología) y que se van a exponer resumidas en el círculo de conversación. Estas lecturas corresponden a varios artículos compilados por Ronny Viales, Jorge Amador y Flora Solano en su libro Concepciones $y$ representaciones de la naturaleza y la ciencia en América Latina (2009), un compendio de investigaciones en el ámbito de la historia de la ciencia y la tecnología que incluye estudios elaborados en México, Costa Rica, Colombia, Paraguay y Argentina, cuya periodización se extiende desde mediados del siglo XIX hasta inicios del siglo XXI que permiten comprender las diferentes etapas del desarrollo de la actividad científica y tecnológica en la región latinoamericana.

Las lecturas escogidas fueron las siguientes, seis en total -una para cada subgrupo de investigación- que representan momentos diferentes del desarrollo histórico de la ciencia y la tecnología en América Latina, cuya discusión permite que el estudiantado comprenda varios de los procesos involucrados que hicieron posible el desarrollo de una actividad científica y tecnológica en la región desde mediados del siglo XIX hasta el presente:

1. Luz Fernanda Azuela: "Geografia e historia natural en la exploración del territorio mexicano". 2. Ronny Viales y Patricia Clare: "El Estado, lo transnacional y la construcción de comunidades científicas"

3. Ronald Díaz: "Estado, comunidades científicas y exploraciones geográficas".

4. Celina Lértora: "La revista Physis y las Ciencias Naturales en Argentina".

5. Rafael Herrera: "Inconsistencia e incertidumbre: Las políticas de ciencia".

6. Nora Garita y Giselle Bustos: "Un acercamiento al imaginario en torno a la Ciencia".

El profesor orienta a la clase dividiendo en grupos de investigación, los cuales sintetizarán la lectura que previamente les fue asignada por él mediante un sorteo realizado en la clase anterior. Además, pondrá a su disposición las horas de consulta para atender las inquietudes que surjan en la clase durante los días previos a la sesión donde se ejecutará el círculo de conversación. El docente también asume el papel de acompañante, es decir, la persona encargada de "planificar, ejecutar, sintetizar, analizar y evaluar el proceso de formación" durante el desarrollo de la técnica (Anónimo, s.f.).

Para la técnica del círculo de conversación se requiere de piezas de diálogo (Arias, 2012), es decir, instrumentos elaborados para ser utilizados por los y las integrantes de los subgrupos con el propósito de garantizar su participación en el transcurso de la discusión de los textos. En este caso, están constituidas por carteles con imágenes impresas a colores relacionadas con instituciones o experiencias latinoamericanas en el ámbito de la ciencia y la tecnología, con el propósito que se usen para indicar quién quiere tomar la palabra en el momento en que emita un criterio u opinión durante el desarrollo de la técnica y una de ellas la emplea el docente. Las piezas de diálogo seleccionadas ccorresponden a los siguientes aspectos: 
1. Centro Espacial de Kuru en Guayana Francesa.

2. Edificio del Centro Nacional de Investigaciones Científicas (CNIC) en Cuba.

3. Edificio de la Comisión Nacional de Ciencia y Tecnología (CONACYT) en México.

4. Instituto Butantán en Brasil.

5. Observatorio de Arecibo en Puerto Rico.

6. Observatorio de La Silla en Chile.

7. Proyecto Ad Astra Rocket en Costa Rica.

Además, para la ceremonia de apertura y de clausura del círculo de conversación (momentos de arranque y conclusión de la técnica), se presentan dos vídeos relacionados con temas referentes a dos momentos históricos del desarrollo científico y tecnológico de América Latina, uno sobre el final del período colonial y otro más reciente. El primer vídeo empleado se titula "Alexander von Humboldt. Narraciones de la historia de América", disponible en la red social YouTube (Westlake, 2013). La presentación de este vídeo ilustra un aspecto de la historia de la ciencia latinoamericana como lo fueron las expediciones de Humboldt a finales del siglo XVIII e inicios del siglo XIX que permite introducir a la clase en el tema que se va a discutir.

La pregunta generadora de la discusión fue planteada para todos los grupos: ¿Qué importancia han tenido la ciencia y la tecnología en el desarrollo de América Latina? Además, a cada grupo se le asignó una pregunta específica para trabajarla en clase:

1. ¿Qué papel jugaron las exploraciones científicas en la ciencia latinoamericana de la primera mitad del siglo XIX?

2. ¿Qué es el régimen de cientificidad?

3. ¿Qué importancia tuvieron las instituciones científicas en el desarrollo de la ciencia latinoamericana?

4. ¿Qué papel juegan las publicaciones periódicas en la difusión del conocimiento científico producido en América Latina?

5. ¿Cómo se han caracterizado las políticas de ciencia y tecnología a partir de la segunda mitad del siglo XX?

6. ¿Cómo se ha construido el imaginario en torno a la ciencia y tecnología en el presente?

El propósito de estas preguntas específicas es servir de apoyo a la discusión de cada uno de los textos, con el fin de responder la pregunta generadora de toda la dinámica del círculo de conversación. De esta forma, se realiza el abordaje del contenido referente a la experiencia latinoamericana en el ámbito científico y tecnológico desde una perspectiva histórica.

La ceremonia de cierre se basó en la proyección del vídeo "LAReferencia: Visibilizando la ciencia en América Latina" sobre experiencias de una red de intercambio de información científica entre países latinoamericanos constituida en años recientes que se puede ubicar en la red social Youtube (RepositoriosLA, 2013). Este vídeo atestigua el avance que ha experimentado el desarrollo científico latinoamericano a inicios del presente siglo, en el que las redes de información científica permiten el intercambio del conocimiento generado en la región, sirve de cierre al círculo de conversación.

La Tabla 2 ilustra la distribución del tiempo empleado para la realización de la estrategia que debido a las limitaciones de horarios para la clase, comprende una sola ronda de participaciones: 


\section{Tabla 2}

Distribución del tiempo para el círculo de conversación aplicado al contenido "El desafío científico y tecnológico"

Tiempo aproximado

Actividad

(minutos)

\begin{tabular}{|c|c|}
\hline 5 & Bienvenida y presentación de la sesión. \\
\hline 10 & $\begin{array}{l}\text { Explicación del círculo de conversación y del uso de la } \\
\text { pieza del diálogo. }\end{array}$ \\
\hline 5 & $\begin{array}{l}\text { Ceremonia de apertura: Vídeo "Alexander von } \\
\text { Humboldt. Narraciones de la historia de América". }\end{array}$ \\
\hline 15 & $\begin{array}{l}\text { Formación de los subgrupos para la discusión del tema } \\
\text { elegido mediante preguntas definidas en la fase de } \\
\text { preparación para la discusión. }\end{array}$ \\
\hline 10 & Refrigerio. \\
\hline 40 & $\begin{array}{l}\text { Conversación general de los temas discutidos en los } \\
\text { subgrupos. (Se recomienda que al ser seis subgrupos, } \\
\text { cada uno emplee tres minutos para responder las } \\
\text { preguntas asignadas y dos para brindar respuesta a las } \\
\text { generadas por los demás subgrupos). }\end{array}$ \\
\hline 10 & Recapitulación de la actividad (A cargo del docente). \\
\hline 5 & $\begin{array}{l}\text { Ceremonia de cierre: Vídeo "LAReferencia: } \\
\text { Visibilizando la ciencia en América Latina". }\end{array}$ \\
\hline
\end{tabular}

Fuente: Arias, 2012. 
El día en que se aplica la técnica del círculo de conversación, se debe respetar el tiempo acordado para cada una de las diferentes actividades. Se cuenta también con la presencia de un estudiante externo o asistente que anota las participaciones correspondientes a cada uno de los grupos.

Sin mayor demora, se debe dar la bienvenida a la clase, presentar la sesión y explicar en qué consiste la técnica del círculo de conversación, así como la importancia de las piezas de diálogo, para luego dividir la clase en grupos con el fin de dialogar sobre cada una de las lecturas, seguida de un breve refrigerio. Después se hace la ronda de conversación con base en las respuestas hechas a las preguntas que se hicieron y las que fueron surgiendo durante el desarrollo de la dinámica del círculo de conversación.

Las actividades de evaluación de esta técnica se realizan mediante la identificación de:

"referentes o criterios la capacidad para crear confianza, construir relaciones, trabajar el tema y profundizar en las reflexiones. Comprende una evaluación formativa que se da a lo largo de la realización del círculo pues los cuestionamientos, posicionamientos teóricos, argumentaciones y opiniones se ponen en diálogo con todos los participantes" (Anónimo, s.f.).

Además, se realiza un sondeo por medio de unas hojas que reparte el docente donde la clase expresa libremente y en forma anónima, su opinión respecto a la aplicación de la técnica, por lo que se reciben tanto opiniones favorables o críticas relacionadas con sus ventajas como el interés despertado en la clase mediante la construcción compartida del conocimiento, la posibilidad de enfocar el tema visto desde diversas ópticas y la posibilidad de externar experiencias relacionadas con el mismo. No obstante, también se debe reconocer que la aplicación de esta técnica trae sus desventajas, entre ellas la necesidad de más tiempo para desarrollar las discusiones con más profundidad, las dificultades de organizar el círculo dentro de un aula pequeña y la programación de un refrigerio que en ocasiones favorece la distracción y la dispersión en la clase, por lo que perfectamente podría organizarse al término de la sesión.

\section{Conclusiones}

El historiador Daniel Montero (2006), quien fungió durante muchos años como profesor de la Escuela de Estudios Generales, al describir su propia experiencia docente desde una perspectiva humanística en el desarrollo de sus clases de Historia de la Cultura, manifestaba que su labor:

"Es un acercamiento y una exteriorización de las experiencias históricas pedagógicas dentro de esa difícil andadura de los estudios humanistas. Proposición utópica, sí, pero realizable. Como utópicos son los estudios humanistas en el siglo XXI, con profundos contenidos éticos y estéticos que coadyuvan al desarrollo integral y armónico del ser humano que ingresa a ellos" (p. 42).

Es precisamente que los y las docentes deben procurar la transmisión del conocimiento mediante técnicas novedosas brindando un enfoque humanístico en el desarrollo de los contenidos de los programas de los cursos de Historia de la Cultura.

En términos generales, la aplicación del círculo de conversación permite mantener el interés de la clase en el tema que se discute, le brinda una perspectiva diferente al estudiantado al participar con base en los conocimientos adquiridos mediante la lectura de los textos asignados. La aplicación de esta técnica garantiza una completa libertad para expresar aspectos relacionados con otros elementos que se consideran importantes respecto a los temas que se debaten y pueden discutirse en el marco de dicha técnica, entre ellos el conocimiento sobre aspectos científicos latinoamericanos vistos en otros cursos o sus experiencias al participar en proyectos o ferias científicas. 
De esta forma, la discusión se enriquece mediante cada una de las participaciones en las que se trata de mantener la secuencia lógica de los artículos, en los que cada grupo toma la palabra mediante las piezas de diálogo donde uno o más integrantes participan. Al mismo tiempo, el docente hace uso también de la palabra a través de una pieza de diálogo, genera nuevas preguntas y la posibilidad de réplicas del resto de la clase.

Por medio de esta técnica, se genera una conversación que evidencia la importancia que ha tenido el desarrollo de la ciencia y la tecnología para los países latinoamericanos y cómo desde el siglo XIX se ha tratado de constituir una cultura científica en la región mediante expediciones científicas, la fundación de instituciones relacionadas con la investigación en distintos campos de la ciencia, la difusión del conocimiento científico a través de publicaciones periódicas y el apoyo del Estado mediante el establecimiento de organismos públicos dedicados al fomento tecno-científico. Todo este proceso ha derivado en el surgimiento de una actividad científica propia en América Latina que se ha visto condicionada por factores de índole política y económica, además que ha tratado de brindar respuestas a los problemas y desafíos de la región.

La valoración de esta experiencia docente se hace tomando en cuenta los alcances y las limitaciones de la misma a partir del análisis de las observaciones hechas durante el círculo de conversación y los reportes escritos hechos por cada uno de los grupos en que se ha dividido la clase. Al término de la sesión se aplica un sondeo, en el que se indica a los y las estudiantes anotar lo que pensaban de la experiencia realizada, del conocimiento aprendido en forma compartida, la relación con los demás integrantes de la clase, así como las ventajas y desventajas experimentadas durante su ejecución.

En términos generales, la aplicación de la técnica del círculo de conversación ha tenido un resultado bastante satisfactorio para desarrollar el contenido de "El desafío científico y tecnológico", ya que se da un consenso en la clase al considerar que constituye una experiencia novedosa y a la vez, más interactiva que otras formas clásicas de transmisión del conocimiento en los cursos de Historia de la Cultura. Esta técnica podría aplicarse para el desarrollo de otros contenidos que también se incluyen en el programa del curso, con el fin de impulsar estrategias didácticas alternativas a las tradicionales lecciones magistrales.

\section{Agradecimiento}

La presente investigación se presentó como ponencia en el marco del I Congreso Universitario de Estudios Humanísticos y VII Congreso de Pensamiento Humanista Regional reunidos en la Escuela de Estudios Generales de la Universidad de Costa Rica, del 3 al 5 de agosto de 2016. El autor agradece a la Comisión Coordinadora del Congreso por autorizar la presentación de este trabajo en dicho evento académico, así como al Programa de Estudios Sociales de la Ciencia, la Técnica y el Medio Ambiente (PESCTMA) del Centro de Investigaciones Geofísicas (CIGEFI) de la Universidad de Costa Rica, por su apoyo en la realización de dicha investigación y al Prof. Luis Omar Mora Sandí por las observaciones hechas en la ejecución de la estrategia didáctica descrita en este artículo. 


\section{Referencias}

Anónimo (s.f.). "Técnicas didácticas. El círculo de conversación”. Universidad de Costa Rica, Facultad de Educación, Escuela de Formación Docente, Departamento de Didáctica Universitaria.

Arias, M. (2012). El círculo de conversación como estrategia didáctica: Una experiencia para reflexionar y aplicar en educación superior. Revista Electrónica Educare, 16(2), 9-24. Recuperado de http://www.redalyc.org/articulo.oa?id=194124286002.

Azuela, L. F. (1995). La institucionalización de la meteorología en México a finales del siglo XIX. En M. L. Rodríguez-Sala y J. O. Moncada (Eds.), La cultura científico-tecnológica en México: Nuevos materiales multidisciplinarios (pp. 99-105). México: UNAM - Instituto de Investigaciones Sociales.

Azuela, L. F. (1996a). La institucionalización de las ciencias en México durante el porfiriato. En M. L. Rodríguez-Sala e I. Guevara (Eds.). Tres etapas del desarrollo de la cultura científico-tecnológica en México (pp. 73-84). México: UNAM.

Azuela, L. F. (1996b). Tres sociedades científicas en el Porfiriato. México: UNAM.

Azuela, L. F. y Guevara, R. (1998). Las relaciones entre la comunidad científica y el poder político en México en el siglo XIX a través del estudio de los farmacéuticos. En P. Aceves (Coord.), Construyendo las ciencias químicas y biológicas (pp. 239-258). México: UAM-X.

Azuela, L. F. y Saldaña, J. J. (1994). De amateurs a profesionales. Las sociedades científicas en México en el siglo XIX. Quipu, 11(2), 135-172.

D’Ambrosio, U. (1986). A Institucionalização do Ciência nos Séculos XIX-XX como Estratégia de Imperialismo Cultural e de Fixação do Poder do Estado os Estudos de Lewis Pyenson e de Harry W. Paul. Quipu, 3(3), 401-417.

Díaz, R. E. (2003). El proceso de institucionalización de la meteorología en Costa Rica (1887-1949) (Tesis de Licenciatura). Universidad de Costa Rica.

Hamburguer, Á. A. (2014). El Socialismo del Siglo XXI en América Latina: Características, desarrollos y desafíos. Revista de Relaciones Internacionales, Estrategia y Seguridad, 9(1), 131-154.

Lafuente, A. y López-Ocón, L. (1996). Tradiciones científicas y expediciones ilustradas en la América hispana del siglo XVII. En J. J. Saldaña (Comp.), La historia social de las ciencias en América latina (pp. 247-281). México: Porrúa.

Margariti, A. (2016). Política monetaria y fiscal en el gobierno de Macri. Recuperado de https://grupojovenfl.files.wordpress.com/2016/07/politicas-monetaria-y-fiscal-de-macrimargariti.pdf.

Martínez, R. y Soto, E. (2012). El Consenso de Washington: la instauración de las políticas neoliberales en América Latina. Política y cultura, 37, 35-64.

Medina, E. (1983). La polémica internalismo / externalismo en la Historia y la Sociología de la Ciencia. Reis: Revista Española de Investigaciones Sociológicas 23: 53-76. 
Montero, D. (2006). Un acercamiento al curso de Historia de la Cultura desde una perspectiva humanística. San José, Costa Rica: EUCR.

RepositoriosLA (2013, diciembre 10). LAReferencia: visibilizando la ciencia en América Latina [Archivo de video]. Recuperado de https://www.youtube.com/watch?v=TtMLHdUQ5JQ.

Rodríguez-Sala, M. L. (2002a). Exploraciones en Baja y Alta California, 1769-1775. Escenarios y personajes. México: UNAM-AMATEditorial.

Rodríguez-Sala, M. L. (2002b). Letrados y técnicos de los siglos XVI y XVII. Escenarios y personajes en la construcción de la actividad científica y técnica novohispana. México: UNAM-IIS.

Rodríguez-Sala, M. L. (Coord.) (2004). Del estamento ocupacional a la comunidad científica: astrónomos-astrólogos e ingenieros (siglo XVII al XIX). México: UNAM.

Saldaña, J. J. (1996a). Ciencia y felicidad pública en la Ilustración americana. En J. J. Saldaña (Comp.), La historia social de las ciencias en América Latina (pp. 151-207). México: Porrúa.

Saldaña, J. J. (1996b). Teatro científico americano. Geografía y cultura en la historiografía latinoamericana de la Ciencia. En J. J Saldaña (Comp.), La historia social de las ciencias en América Latina (pp. 7-41). México: Porrúa.

Solano, F. J. (1999). El proceso de institucionalización de la meteorología en Costa Rica en el siglo XIX (Tesis de Licenciatura). Universidad de Costa Rica.

Vessuri, H. M. C. (1996). La ciencia académica en América Latina en el siglo XX. En J. J. Saldaña (Comp.), La historia social de las ciencias en América Latina (pp. 437-479). México: Porrúa.

Valdivia, Ó. (1996). Matemáticas y astronomía precolombinas. En J. J. Saldaña (Comp.), La historia social de las ciencias en América Latina (pp. 91-118). México: Porrúa.

Viales, R., Amador, J. A. y Solano, F. J. (Eds.) (2009). Concepciones y representaciones de la naturaleza y la ciencia en América Latina. San José: Universidad de Costa Rica, Vicerrectoría de Investigación.

Viales, R. y Clare, P. (2006-2007). El Estado, lo transnacional y la construcción de comunidades científicas en la Costa Rica liberal (1870-1930). La construcción de un "régimen de cientificidad". Diálogos. Revista Electrónica de Historia, 6(2), 145-168. Recuperado de http://www.ts.ucr.ac.cr/ historia/articulos/2006/vol2/6-rviales clare.pdf.

Viales, R. y Clare, P. (2009). El Estado, lo transnacional y la construcción de comunidades científicas en la Costa Rica liberal (1870-1930). La construcción de un "régimen de cientificidad". En R. Viales, J. A. Amador y F. J. Solano (Eds.), Concepciones y representaciones de la naturaleza y la ciencia en América Latina (pp. 97-109). San José: Universidad de Costa Rica, Vicerrectoría de Investigación.

Westlake, D. (2013, febrero 16). Alexander von Humboldt. Narraciones de la Historia de América [Archivo de video]. Recuperado de https://www.youtube.com/watch?v=1DtOYMnMU2I. 
Ciencia y tecnología en América...

\section{¿Cómo citar este artículo?}

Díaz Bolaños, R. (2017). Ciencia y tecnología en América Latina: Una aproximación desde la Historia de la Cultura. Revista humanidades, 7(2). doi: http://dx.doi.org/10.15517/h.v7i2.29579 